\title{
Techniques to improve the limited degree of freedom inherent from laparoscopic surgery during laparoscopic duct-to-mucosa pancreaticojejunostomy
}

\author{
Boram LEE, Yoo-Seok YOON*, Ho-Seong HAN, Jai Young CHO, Hae Won LEE, Jun Suh LEE, Moonhwan KIM
}

Department of Surgery, Seoul National University Bundang Hospital, Seongnam, Korea

Introduction: Although an increasing number of reports have shown favorable outcomes of laparoscopic pancreaticoduodenectomy (LPD), the penetration rate of LPD is still low due to concerns about complex procedure. Particularly laparoscopic pancreatojejunostomy (PJ) is a highly technical demanding procedure due to reduced degree of freedom inherent to laparoscopic instruments. Here we describe our technique for overcoming technical difficulties of laparoscopic PJ.

Methods: Six trocars are placed: four trocars on both sides of the upper abdomen in a curvilinear shape with the infra-umbilical trocar in the center and one 12-mm trocar in the epigastrium. The operator stands between both legs of the patient and the laparoscopic camera is introduced through the right lower trocar.

Results: We perform a two-layer, duct-to-mucosa PJ in an end-to-side fashion. For the outer layer anastomosis between the pancreas parenchyma and jejunum, the left lower trocar which is in alignment with the pancreatic transection line is used for the needle to be placed perpendicular to the anastomosis line. For the duct-to-mucosa anastomosis, three trocars (left lower, left upper, umbilical) is alternatively used to make various angles of suture. This technique increses the degree of freedom of the straight laparoscopic needle holder when only one trocar is used for duct-to-mucosa anastomosis.

Conclusions: The trocar position perpendicular to the anastomosis line and alternatve use of multiple trocars is useful to encompass the 360-degree anastomosis for laparoscopic duct-to-mucosa PJ. 